\title{
Synoviolin inhibitor LS-102 reduces endoplasmic reticulum stress-induced collagen secretion in an in vitro model of stress-related interstitial pneumonia
}

\author{
FUKAMI NAKAJIMA $^{1,2}$, SATOKO ARATANI ${ }^{2,3}$, HIDETOSHI FUJITA ${ }^{2,3}$, NAOKO YAGISHITA ${ }^{4}$, \\ SHIZUKO ICHINOSE $^{5}$, KOSHI MAKITA ${ }^{1}$, YASUHIRO SETOGUCHI ${ }^{6}$ and TOSHIHIRO NAKAJIMA ${ }^{2,3,7}$
}

\author{
${ }^{1}$ Department of Anesthesiology, Graduate School of Medical and Dental Sciences, Tokyo Medical and Dental University, \\ Bunkyo-ku, Tokyo 113-8519; ${ }^{2}$ Department of Locomotor Science, Institute of Medical Science, and \\ ${ }^{3}$ Future Medical Science, Institute of Medical Science, Tokyo Medical University, Shinjuku-ku, Tokyo 160-8402; \\ ${ }^{4}$ Institute of Medical Science, St. Marianna University School of Medicine, Kawasaki, Kanagawa 216-8512; \\ ${ }^{5}$ Research Center for Medical and Dental Science, Tokyo Medical and Dental University, Bunkyo-ku, \\ Tokyo 113-8510; ${ }^{6}$ Department of Respiratory Medicine, and ${ }^{7}$ Integrated Genome Editing Section (iGES), \\ Tokyo Medical University, Shinjuku-ku, Tokyo 160-8402, Japan
}

Received July 4, 2014; Accepted October 10, 2014

DOI: $10.3892 /$ ijmm.2014.1984

\begin{abstract}
The deletion mutation of exon 4 in surfactant protein C (SP-C), a lung surfactant protein, has been identified in parent-child cases of familial interstitial pneumonia. It has been shown that this mutation induces endoplasmic reticulum (ER) stress. Synoviolin is an E3 ubiquitin ligase that is localized to the ER and is an important factor in the degradation of ER-related proteins. It has been demonstrated that synoviolin is involved in liver fibrosis. In the present study, we investigated the involvement of synoviolin in the pathogenesis of interstitial pneumonia caused by the exon 4 deletion in the SP-C gene. We transfected wild-type and exon 4-deleted SP-C genes into A549 human lung adenocarcinoma cells and measured the secretion of collagen, which is a representative extracellular matrix protein involved in fibrosis. Secreted collagen levels were increased in the culture medium in SP-C mutants compared to the wild-type cells. Furthermore, the transcription of mRNAs coding for factors associated with fibrosis was increased. Subsequently, to assess the involvement of synoviolin, we constructed plasmids with a luciferase gene under the control of the synoviolin promoter. The A549 cells were transfected with the construct along with the exon 4-deleted SP-C plasmid for use in the luciferase assay. We found a 1.6-fold increase in luciferase activaty in the cells carrying exon 4 deleted SP-C, as well as an increase in intrinsic synoviolin expression at the mRNA and protein levels. Collagen
\end{abstract}

Correspondence to: Professor Toshihiro Nakajima, Department of Locomotor Science, Tokyo Medical University, 6-1-1 Shinjuku, Shinjuku-ku, Tokyo 160-8402, Japan

E-mail: marlin@tokyo-med.ac.jp

Key words: endoplasmic reticulum stress, fibrosis, E3 ubiquitin ligase synoviolin secretion was decreased by the addition of LS-102, a synoviolin inhibitor, to the A549 culture medium following transfection with wild-type and exon 4-deleted SP-C. These results demonstrate that synoviolin is involved in the onset of interstitial pneumonia induced by exon 4-deleted SP-C, which suggests that synoviolin inhibitors may be used in the treatment of the disease.

\section{Introduction}

Idiopathic pulmonary fibrosis (IPF) is part of a large group of diffuse parenchymal lung diseases, that are characterized by progressive parenchymal fibrosis, without any known cause and poor prognosis. Nogee et al conducted a genetic analysis of a mother (onset age, 1 year) with desquamative interstitial pneumonia (DIP) and a child (onset age, 6 weeks) with non-specific interstitial pneumonia (NSIP), and identified a heterozygous mutation of the surfactant protein $\mathrm{C}$ (SP-C) gene (deletion of exon 4, SP-C ${ }^{\Delta \operatorname{exon} 4}$ ) for the first time (1). SP-C is a component of the pulmonary surfactant, which is a heterogeneous complex of lipids and proteins that lowers lung-surface tension and contributes to the host defense system. The SP-C gene is located on the short arm of chromosome 8, and comprises 6 exons and 5 introns (2). The precursor of SP-C, proSP-C, consists of 197 amino acids and is transformed into mature SP-C (consisting of 24-58 amino acids) through intracellular cleavage and modification $(3,4)$. ProSP-C $\mathrm{C}^{\Delta \text { exon4 }}$ has been found to induce endoplasmic reticulum (ER) stress $(5,6)$.

Fibrosis is defined by the accumulation of excess extracellular matrix (ECM) components, such as collagen, in and around inflamed or damaged tissue $(7,8)$. Fibrosis can develop in the majority of tissue types, and advanced fibrosis causes fibrotic diseases, such as pulmonary fibrosis, liver cirrhosis and renal fibrosis, resulting in organ dysfunction and eventually death. According to recent studies, synoviolin/HRD1 is involved in liver fibrosis (9) and collagen synthesis in the kidneys (10). Synoviolin, 
which we have previously cloned (11), is an ER-resident E3 ubiquitin ligase with a RING finger domain that activates the ER-associated degradation (ERAD) pathway (11). Synoviolin is ubiquitously expressed in all organs of the body, particularly in the liver, pancreas and skeletal muscle (12). Synoviolin is upregulated in response to ER stress and the overexpression of human inositol-requiring kinase 1 (IRE1) and activating transcription factor 6 (ATF6) (12,13). We have previously demonstrated that heterozygous synoviolin knockout mice are resistant to $\mathrm{CCl}_{4}$-induced liver fibrosis. In addition, we have demonstrated that collagen secretion is reduced by synoviolin deficiency in mouse embryonic fibroblasts, suggesting the involvement of synoviolin in collagen secretion (9). Li et al (10) demonstrated that synoviolin expression was increased in a mouse model of renal interstitial fibrosis caused by unilateral ureteral obstruction (UUO). In addition, they demonstrated that collagen secretion is reduced by synoviolin deletion in NRK-49F cells (normal rat kidney interstitial fibroblasts) (10). These data suggest that synoviolin expression is increased by ER stress and that synoviolin promotes collagen synthesis. Thus, we hypothesized that synoviolin is involved in lung fibrosis, and in particular, in ER stress-related lung fibrosis. In order to confirm our hypothesis, in the present study, we investigated the involvement of synoviolin and synoviolin inhibitor, LS-102, in collagen secretion in an in vitro model of IPF by transfecting A549 cells (a human lung adenocarcinoma epithelial cell line) with SP-C lacking exon 4.

To investigate our hypothesis that synoviolin is involved in lung fibrosis and that synoviolin inhibitors are candidate antifibrotic agents, we investigated the association between synoviolin and collagen secretion in an in vitro model, in which A549 cells were transfected with SP-C $\mathrm{C}^{\Delta \text { exon4}}$, which has been reported to induce ER stress $(5,6)$. In the present study, we did not use any chemical or physical method to induce fibrosis; instead, we focused on an SP-C mutation, SP-C $\mathrm{C}^{\Delta \text { exon } 4}$, which has been identified in parent-child cases of familial interstitial pneumonia (1).

\section{Materials and methods}

Cell culture. The A549 human lung adenocarcinoma cell line was purchased from the American Type Culture Collection (ATCC, Manassas, VA, USA). The A549 cells were cultured in minimum essential medium (Sigma-Aldrich, St. Louis, MO, USA) supplemented with $1 \%$ penicillin, $1 \%$ streptomycin and $10 \%$ (heat-inactivated) fetal bovine serum (FBS).

Transfection of cells with plasmid constructs and siRNA. The wild-type proSP-C expression vector was constructed using pcDNA3-FLAG (Invitrogen, Carlsbad, CA, USA) by the insertion of wild-type proSP-C cDNA that was isolated from a human lung cDNA library (Takara Bio Co., Tokyo, Japan).

SP-C $\mathrm{C}^{\Delta \text { exon4 }}$ (deletion of exon 4) was constructed according to previously published techniques (14). All constructs were confirmed by DNA sequencing. The A549 cells were grown for $24 \mathrm{~h}$ and transfected with wild-type SP-C or mutant SP-C constructs using Lipofectamine 2000 (Invitrogen) as per the manufacturer's instructions.

The sequences of the synoviolin siRNA and scrambled synoviolin siRNA were identical to those described in a previous study (15), and were chemically synthesized at
Hokkaido System Science (Hokkaido, Japan). The A549 cells were seeded at 40-50\% confluency in 6-well plates and cultured for $24 \mathrm{~h}$. Subsequently, $50 \mathrm{nmol}$ of annealed RNA duplex was transfected using Lipofectamine 2000 according to the manufacturer's instructions.

Antibodies. The antibodies used in this study were as follows: anti-synoviolin/HRD1 monoclonal antibody as previously described (11), anti-FLAG antibody (anti-FLAG M2 monoclonal antibody, F3165; Sigma-Aldrich), antihemagglutinin-A (HA) 3F10 monoclonal antibody (1867423; Roche Applied Science) and anti- $\beta$-actin monoclonal antibody (A5441; Sigma-Aldrich).

Preparation of cellular protein. Cell cultures were harvested and proteins were extracted using a cell extraction buffer containing 0.05 M Tris- $\mathrm{HCl}(\mathrm{pH} 8.0), 0.15 \mathrm{M} \mathrm{NaCl}, 5.0 \mathrm{mM}$ ethylenediaminetetraacetic acid (EDTA), 1\% NP-40 and protease inhibitors $(1 \mu \mathrm{g} / \mathrm{ml}$ leupeptin, $1 \mu \mathrm{g} / \mathrm{ml}$ dithiothreitol (DTT), $1 \mu \mathrm{g} / \mathrm{ml}$ pepstatin and $1.5 \mu \mathrm{g} / \mathrm{ml}$ aprotinin) at $4^{\circ} \mathrm{C}$.

Reporter assay. The A549 cells were plated on 24-well plates $\left(5 \times 10^{4}\right.$ cells/well). The synoviolin promoter (SyG-2055/+845 PGV-B2) was prepared as described in a previous study (16). For transient transfection into the A549 cells, $20 \mathrm{ng} /$ well synoviolin promoter and $50 \mathrm{ng} / \mathrm{well}$ test plasmids (SP-C $\mathrm{C}^{1-197}$ or SP-C ${ }^{\Delta \text { exon4}}$ ) were co-transfected using Lipofectamine 2000 (Invitrogen) according to the manufacturer's recommendations. After $24 \mathrm{~h}$, the cultures were aspirated and the cells were added to $100 \mu \mathrm{l}$ of passive lysis buffer (Promega, Madison, WI, USA). The cell debris was pelleted and $5 \mu \mathrm{l}$ of supernatant were collected and immediately analyzed for luciferase activity using the Promega Luciferase assay system (Promega) and a microplate luminometer (Centro XS3 LB 960; Berthold Technologies, Wildbad, Germany). The $5 \mu$ l supernatant samples were used to measure the protein concentration in each well using a multi-spectrophotometer (Viento XS; Dainippon Sumitomo Pharma, Tokyo, Japan). The assay was performed in triplicate and the mean values were calculated for each sample. The luciferase activity results were normalized to the protein concentration in each sample.

Western blot analysis. The A549 cells were seeded at 40-50\% confluency in 100-mm plates and harvested $18 \mathrm{~h}$ following transfection. Tunicamycin-treated $(10 \mu \mathrm{g} / \mathrm{ml})$ A549 cells were prepared as a model of chemical induction of ER stress. Cellular protein extracts were resolved by $10 \%$ SDS-PAGE, transferred onto a nitrocellulose membrane and immunoblotted with anti-synoviolin/HRD1 monoclonal antibodies followed by horseradish peroxidase-conjugated secondary antibodies. The antigen-antibody complexes were visualized using an ECL detection system (Promega). $\beta$-actin expression was used as an internal control. Immunoblots were scanned and band densities were quantified using Image J software.

Real-time polymerase chain reaction (PCR). The A549 cells were grown in $100-\mathrm{mm}$ culture plates. Forty-eight hours following transfection, total RNA from the A549 cells was isolated using Isogen II (Nippon Gene, Tokyo, Japan) and cDNA was synthesized using RiverTra Ace (Toyobo, Tokyo, 

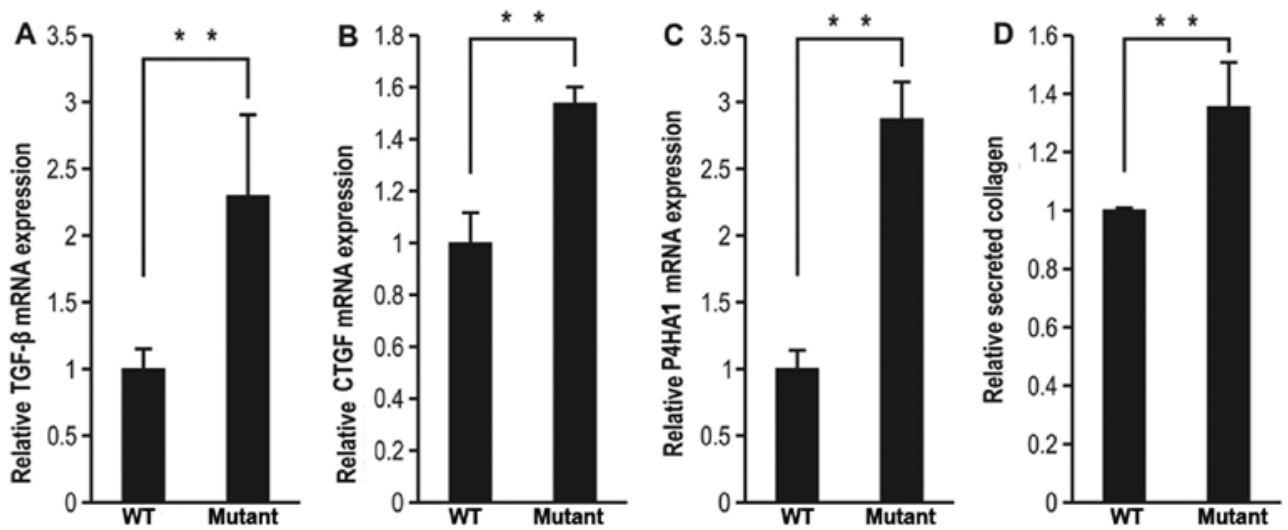

Figure 1. Effect of surfactant protein C (SP-C) overexpression on collagen secretion in A549 cells. The mutant SP-C gene increased the mRNA expression levels of (A) transforming growth factor- $\beta$ (TGF- $\beta$ ), (B) connective tissue growth factor (CTGF) and (C) prolyl 4-hydroxylase subunit alpha 1 (P4HA1) by approximately 2.3-, 1.5- and 2.9-fold, respectively, compared to the wild-type. (D) A549 cells transfected with mutant SP-C secreted an approximately 1.3-fold greater amount of collagen into the medium than the cells transfected with wild-type SP-C. Data are the means \pm standard error of the mean (SEM) of 3 experiments. "** P $<0.01$. WT, wild-type.

Japan) according to the manufacturer's instructions. Real-time PCR relative quantification analysis was performed to measure the mRNA expression levels of transforming growth factor- $\beta$ (TGF- $\beta$ ), connective tissue growth factor (CTGF), prolyl 4-hydroxylase subunit alpha 1 (P4HA1), X-box binding protein 1 (XBP1) and synoviolin using the FastStart Universal Probe Master mix (ROX; Roche Applied Science, Indianapolis, IN, USA), primers from the Universal Probe Library Assay Design Center and probes from the universal probe library set (both from Roche Applied Science, Tokyo, Japan) with the Step One Plus Detection System and software (Applied Biosystems, Life Technologies Japan, Tokyo, Japan). The primers and probes used are as follows: TGF- $\beta$ forward, GCAGCACGTGGAGCTGTA and reverse, CAGCCGGTTGC TGAGGTA (probe: Universal probe library probe \#72); CTGF forward, AGCTGACCTGGAAGAGAACATT and reverse, GCTCGGTATGTCTTCATGCTG (probe: Universal probe library probe \#71); P4HA1 forward, TCGTCAAAGACCTAG CAAAACC and reverse, CCGTCTCCAAGTCTCCTGTTA (probe: Universal probe library probe \#30); XBP1 forward, GGAGTTAAGACAGCGCTTGG and reverse, CACTGG CCTCACTTCATTCC (probe: Universal probe library probe \#37); synoviolin forward, TTCGTCAGCCACGCCTAT and reverse, GAGCACCATCGTCATCAGG (probe: Universal probe library probe \#89). The protocol included 1 cycle at $95^{\circ} \mathrm{C}$ for $10 \mathrm{~min}$, followed by 40 cycles at $95^{\circ} \mathrm{C}$ for $1 \mathrm{sec}$ and $60^{\circ} \mathrm{C}$ for $20 \mathrm{sec}$, according to the manufacturer's recommendations. The mRNA level was normalized relative to the amount of the transcript coding for $18 \mathrm{~S}$ rRNA.

Measurement of soluble secreted collagen. The A549 cells were seeded at $40-50 \%$ confluency in 6-well plates. Twelve hours following transfection, the medium was replaced with serumfree Opti-MEM (Gibco, Life Technologies Japan, Tokyo, Japan) to eliminate the effects of serum on collagen measurement. At $30 \mathrm{~h}$ following transfection, the total soluble collagen in the culture supernatants was measured using the Sircol collagen assay method (Biocolor, County Antrim, UK) according to the manufacturer's instructions. The assay was performed in triplicate and the mean values of each sample were calculated.
Immunoprecipitation. The cells were plated on 100-mm tissue culture dishes and MG132 was added at a concentration of $20 \mu \mathrm{M} 24 \mathrm{~h}$ following transfection. The cells were co-transfected with hemagglutinin (HA)-tagged ubiquitin (Ub) and an SP-C wild-type, SP-C mutant, or empty vector. Co-transfected with the synoviolin gene were also prepared. Twenty-seven hours following transfection, the cells were harvested and lysed with $200 \mu 1$ of lysis buffer [50 mM HEPES-KOH (pH 7.9), $150 \mathrm{mM} \mathrm{KCl,} \mathrm{1 \%} \mathrm{Triton} \mathrm{X-100,}$ $1 \mathrm{mM}$ phenylmethylsulfonyl fluoride (PMSF), 10\% glycerol, $2 \mu \mathrm{g} / \mathrm{ml}$ aprotinin, $2 \mu \mathrm{g} / \mathrm{ml}$ leupeptin, $2 \mu \mathrm{g} / \mathrm{ml}$ pepstatin A and $20 \mu \mathrm{M}$ MG132 (a proteasome inhibitor)]. Following centrifugation at $5,000 \mathrm{rpm}$ for $10 \mathrm{~min}, 150 \mu \mathrm{l}$ of the clear supernatant was incubated with $5 \mu \mathrm{l}$ of normal mouse $\mathrm{IgG}$ (Santa Cruz Biotechnology, Inc., Santa Cruz, CA, USA), $30 \mu 1$ of protein G-Sepharose (GE Healthcare, Tokyo, Japan) and $350 \mu \mathrm{l}$ of lysis buffer at $4^{\circ} \mathrm{C}$ with rotation for $30 \mathrm{~min}$. The supernatant was incubated with $2 \mu \mathrm{l}$ of anti-FLAG M2 monoclonal antibody at $4^{\circ} \mathrm{C}$ with rotation for $2 \mathrm{~h}$, after which $40 \mu \mathrm{l}$ of protein $\mathrm{G}$ were added, followed by incubation at $4^{\circ} \mathrm{C}$ with rotation for $1 \mathrm{~h}$. The immunoprecipitates were solubilized in SDS sample buffer [0.078 M Tris- $\mathrm{HCl}$ (pH 6.8), 2\% SDS, $10 \%$ glycerol, $5 \%$ 2-mercaptoethanol and $0.003 \%$ bromophenol blue] and then subjected to $4-15 \%$ gradient SDS-PAGE (Bio-Rad, Hercules, CA, USA).

Synoviolin inhibitor, LS-102. Synoviolin inhibitor, LS-102, was identified by screening approximately 4 million compounds for potential synoviolin enzyme inhibitors as described in a previous study (5). Dimethyl sulfoxide (DMSO) is used as solvent for LS-102; thus, we used DMSO as a control. Twelve hours following transfection, Opti-MEM (no serum added) was replaced, and LS-102 $(5 \mu \mathrm{M})$ or DMSO $(5 \mu \mathrm{M})$ was added to the medium to evaluate the effect of LS-102.

Statistical analysis. All data are expressed as the means \pm standard deviation (SD). Differences between groups were examined using the Student's t-test and considered statistically significant at $\mathrm{P}<0.05$. All results were derived from at least 3 independent experiments. 

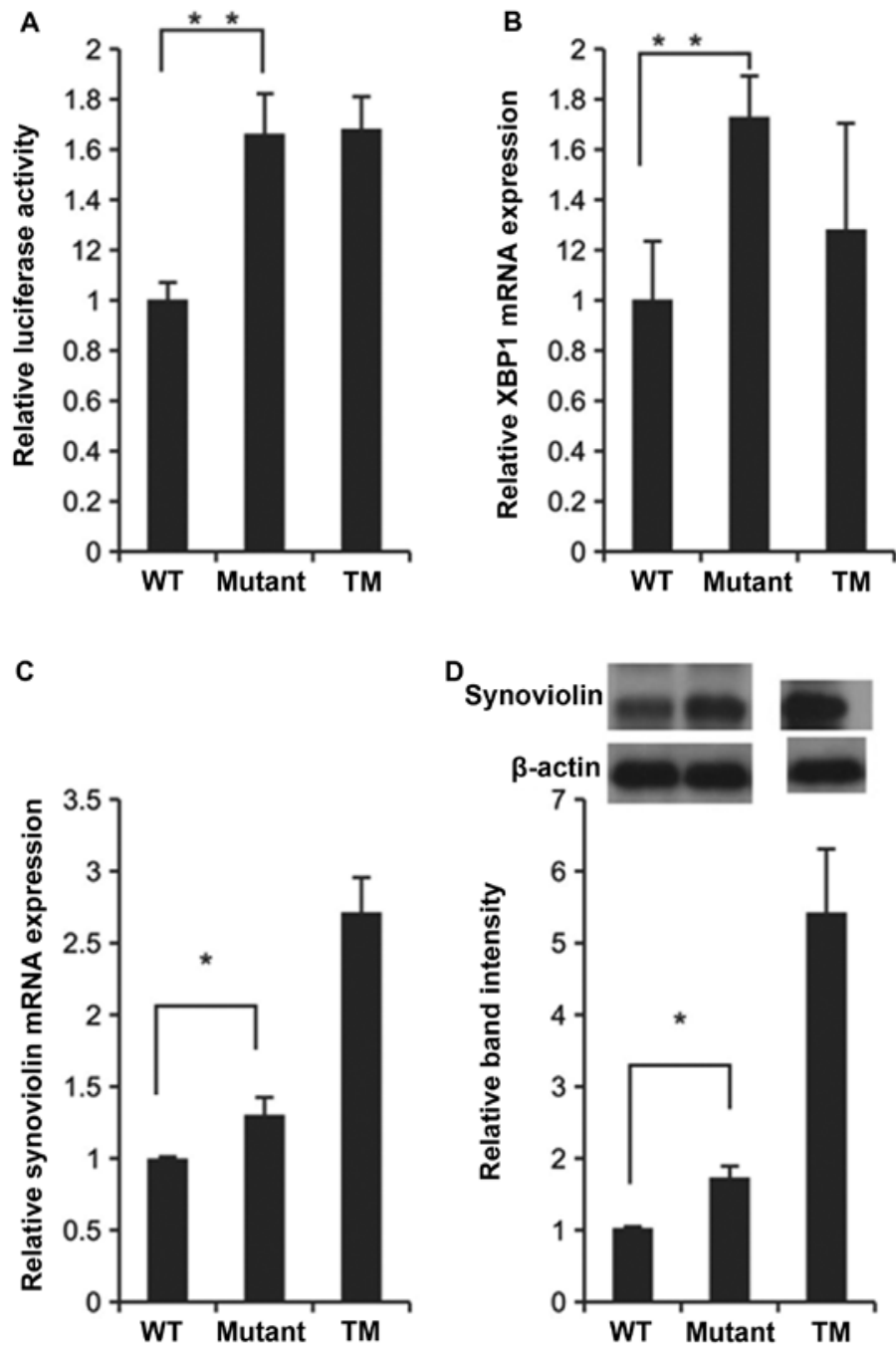

Figure 2. Effect of surfactant protein C (SP-C) $)^{\Delta x o n 4}$ overexpression on synoviolin expression in A549 cells. (A) Luciferase assay revealed that the A549 cells expressing mutant SP-C showed a 1.6-fold higher luciferase activity than the wild-type SP-C-expressing cells. The mRNA expression levels of (B) X-box binding protein 1 (XBP1) and (C) synoviolin were increased in the mutant SP-C-expressing A549 cells compared with the wild-type SP-C-expressing cells. (D) Transfection of A549 cells with mutant SP-C increased the expression of synoviolin protein compared with the cells transfected with wild-type SP-C. Data are the means \pm standard error of the mean (SEM) of 3 experiments. WT, wild-type; TM, tunicamycin [positive control of endoplasmic reticulum (ER) stress]. ${ }^{*} \mathrm{P}<0.05$ and ${ }^{* *} \mathrm{P}<0.01$.

\section{Results}

Overexpression of SP-C $C^{\Delta e x o n 4}$ promotes collagen secretion in A549 cells. Studies have found an association between mutations in the SP-C gene and familial pulmonary fibrosis $(1,17,18)$. Therefore, in this study we focused on the SP-C $\mathrm{C}^{\text {exon4 }}$ mutation. To assess the association between the SP-C ${ }^{\Delta \text { exon } 4}$ mutation and fibrosis in the current experimental system, we measured the mRNA expression levels of transforming growth factor- $\beta$ (TGF- $\beta$ ), connective tissue growth factor (CTGF) and prolyl 4-hydroxylase subunit alpha 1 (P4HA1), which are responsible for enhancing the production of collagen protein, a typical ECM component involved in fibrosis (19-21). Transfection of the cells with the mutant SP-C gene increased the mRNA expression levels of TGF- $\beta$, CTGF and P4HA1 by approximately 2.3-, 1.5and 2.9-fold, respectively, compared to the wild-type (Fig. 1A-C).

Fibrosis is attributed to the excess deposition of ECM components in and around inflamed or damaged tissue (8). Collagen is a major component of the ECM; therefore, we measured collagen secretion following transfection of the cells with mutant SP-C.
To confirm that mutant SP-C promoted collagen secretion from mutant SP-C-expressing A549 cells, collagen secretion was measured following transfection with a wild-type or mutant SP-C gene. The A549 cells transfected with the mutant SP-C gene secreted approximately 1.3-fold more collagen into the medium than the cells transfected with wild-type SP-C (Fig. 1D).

$S P-C^{\Delta e x o n 4}$ increases the expression of synoviolin. To examine the hypothesis that synoviolin plays a role in fibrosis induced by SP-C $\mathrm{C}^{\Delta \text { exon4}}$, we evaluated the level of synoviolin expression in SP-C $\mathrm{C}^{\mathrm{Aexon} 4}$-expressing A549 cells. First, we constructed a plasmid carrying a luciferase gene with expression regulated by a synoviolin promoter, which was co-transfected together with the wild-type SP-C or mutant SP-C gene expression plasmid into the A549 cells. Twenty-four hours following transfection, a luciferase assay was conducted. The A549 cells expressing mutant SP-C showed 1.6-fold greater luciferase activity than the wild-type SP-C-expressing cells (Fig. 2A). These data suggest that the transcriptional activity of synoviolin was enhanced in the SP-C ${ }^{\Delta \text { exon4 }}$ gene-transfected cells. 

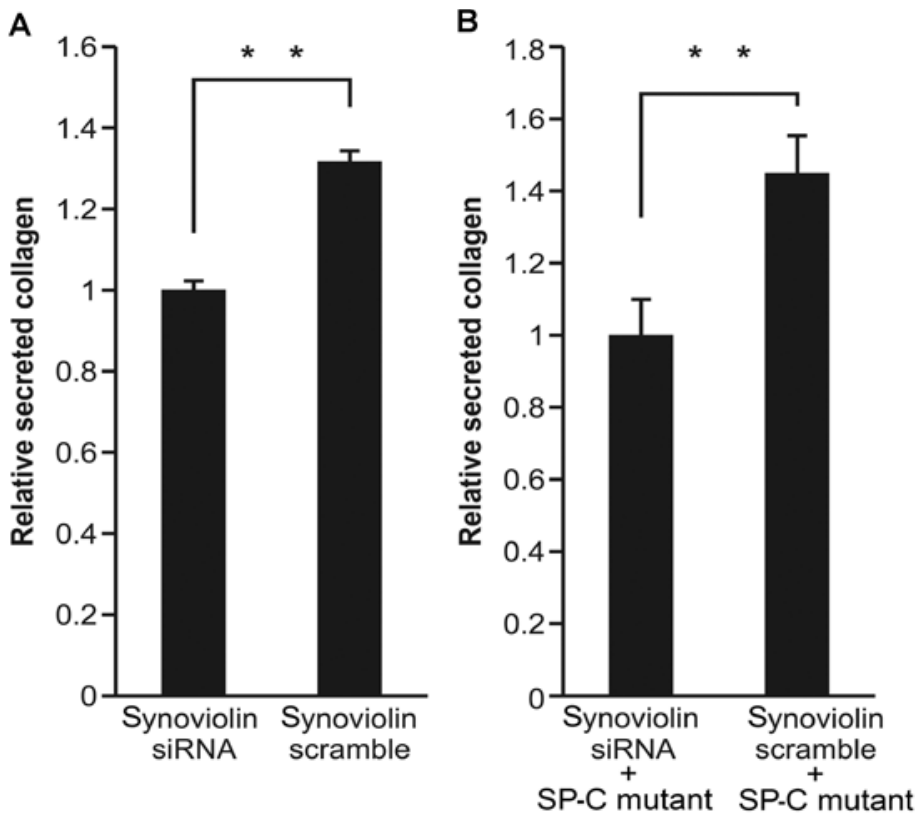

Figure 3. Effect of synoviolin siRNA on synoviolin secretion in A549 cells transfected with surfactant protein C (SP-C) ${ }^{\Delta e x o n 4}$. (A) Collagen secretion was decreased by synoviolin siRNA in A549 cells. (B) A549 cells were co-transfected with SP-C $\mathrm{C}^{\Delta e x o n 4}$ and synoviolin siRNA or scrambled siRNA. The amount of soluble secreted collagen in the supernatant of synoviolin siRNA-transfected SP-C $\mathrm{C}^{\Delta e x n 4}$-expressing A549 cells was less than that produced by scrambled siRNAtransfected SP-C ${ }^{\Delta e x o n 4}$-expressing A549 cells. Data are the means \pm standard error of the mean (SEM) of 3 experiments. ${ }^{* *} \mathrm{P}<0.01$.

Subsequently, to confirm that ER stress is induced in this experimental systemand that the mRNA expression of synoviolin is activated in the mutant SP-C-transfected cells, we examined the expression levels of X-box binding protein 1 (XBP1) and synoviolin in the A549 cells by real-time PCR. The mRNA expression levels of XBP1 and synoviolin were increased in the mutant SP-C-expressing A549 cells in comparison to the wild-type SP-C-expressing cells (Fig. 2B and C).

Finally, to assess the protein expression of synoviolin, we performed western blot analysis with an anti-synoviolin antibody. Transfection of the A549 cells with mutant SP-C increased the protein expression of synoviolin compared with the cells transfected with wild-type SP-C (Fig. 2D). Thus, the overexpression of SP-C ${ }^{\Delta \text { exon4 }}$ in the A549 cells increased the transcriptional activity of synoviolin and the expression of synoviolin at the mRNA and protein levels.

Synoviolin knockdown decreases collagen secretion in A549 cells transfected with SP-C $C^{4 \text { exon } 4}$. To explore the role of synoviolin in collagen secretion in the A549 cells, we evaluated collagen production in A549 cells following transfection with siRNA targeting synoviolin. We found that the level of soluble secreted collagen in the supernatant of A549 cells after the knockdown of synoviolin was approximately $20 \%$ less than that produced by the scrambled siRNA-transfected A549 cells (Fig. 3A). Furthermore, we analyzed the effects of synoviolin siRNA on collagen secretion in SP-C $\mathrm{C}^{\Delta \text { exon}_{4}}$ expressing A549 cells. The A549 cells were co-transfected with SP-C ${ }^{\Delta \mathrm{exon} 4}$ and either synoviolin siRNA or scrambled siRNA. The amount of soluble secreted collagen in the supernatant of the synoviolin siRNA-transfected SP-C $\mathrm{C}^{\Delta \text { exon4 }}$-expressing A549 cells was approximately $30 \%$ less than that produced by scrambled siRNA-transfected SP-C ${ }^{\text {sexon4}}$-expressing A549 cells (Fig. 3B).
Synoviolin ubiquitinates proSP-C $C^{4 \text { exon } 4}$ as a substrate. To demonstrate the ubiquitination of proSP-C ${ }^{\operatorname{exon} 4}$, the A549 cells were co-transfected with hemagglutinin (HA)-tagged ubiquitin (Ub) and an SP-C wild-type, SP-C mutant, or empty vector. In addition, to determine whether synoviolin ubiquitinates proSP-C ${ }^{\Delta e x o n 4}$ as a substrate, cells co-transfected with the synoviolin gene were also prepared. Following immunoprecipitation with an anti-FLAG antibody, western blot analysis was performed using an anti-HA antibody. The cells transfected with SP-C $\mathrm{C}^{\Delta \text { exon4 }}$ showed a ladder pattern, suggesting ubiquitination. The synoviolin-containing cells showed a more intense band (Fig. 4). These results suggested that the proSP-C $\mathrm{C}^{\Delta \text { exon4 }}$ protein was ubiquitinated in this experimental system and that synoviolin may participate in this ubiquitination.

Collagen secretion is suppressed by the synoviolin inhibitor, $L S-102$. To investigate the effects of synoviolin inhibition on collagen secretion in A549 cells, we performed experiments using the synoviolin inhibitor, LS-102, as described in a previous study of ours (22), in which we identified LS-102 by screening approximately 4 million compounds for potential synoviolin enzyme inhibitors. As a result, LS-101 and LS-102 were identified as compounds that inhibited the autoubiquitination activity of synoviolin. These compounds were administered in a mouse model of collagen-induced arthritis, in which they demonstrated protective effects against arthritis. LS-101 had more potent inhibitory effects on the synoviolin enzyme than LS-102. However, LS-102 was used in this study as it is more selective for synoviolin than LS-101. Twelve hours following transfection, Opti-MEM (no serum added) was replaced, and LS-102 or DMSO ( $5 \mu \mathrm{M}$; as a control) was added to the medium. The tunicamycin $(10 \mu \mathrm{g} / \mathrm{ml})$-treated A549 cells were also prepared as a positive control for ER stress induction. LS-102 significantly decreased collagen secretion into the medium (Fig. 5). 


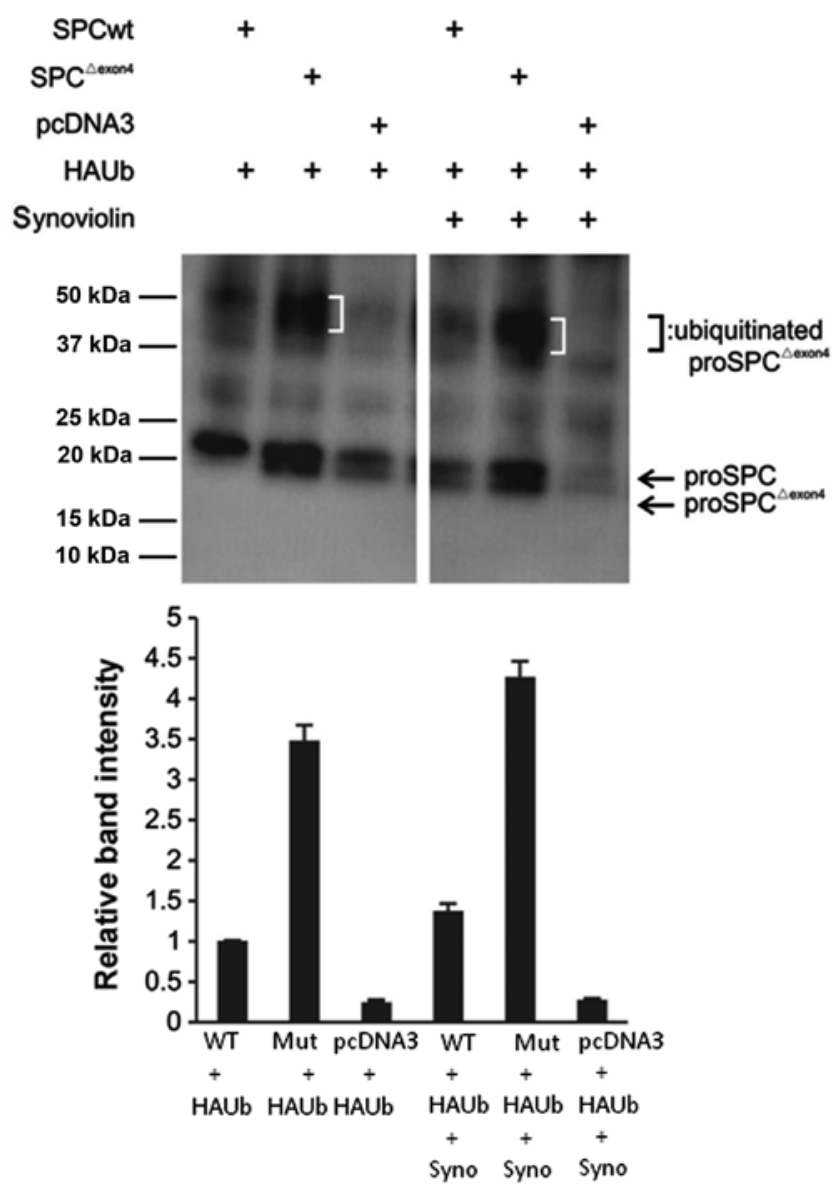

Figure 4. Ubiquitination of precursor of surfactant protein C (proSP-C) ${ }^{\Delta e x o n 4}$. A549 cells were co-transfected with hemagglutinin (HA)-tagged ubiquitin (Ub) and SP-C wild-type, SP-C mutant, or an empty vector. In addition, samples were also prepared that were co-transfected with the synoviolin gene. After immunoprecipitation with an anti-FLAG antibody, western blot analysis was performed using an anti-HA antibody. The cells transfected with SP-C ${ }^{\Delta e x o n} 4$ showed a ladder pattern suggesting ubiquitination. The synoviolin-containing cells showed a more intense band. Data are the means \pm standard error of the mean (SEM) of 3 experiments. Syno, synoviolin.

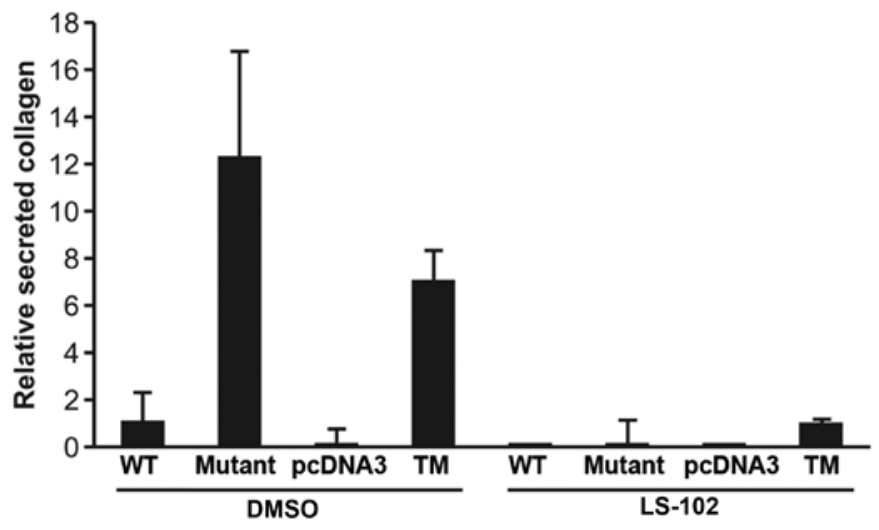

Figure 5. Effect of the synoviolin inhibitor, LS-102, on collagen secretion in A549 cells. LS-102 significantly decreased collagen secretion into the medium. Data are the means \pm standard error of the mean (SEM) of 3 experiments. WT, wild-type; TM, Tunicamycin [positive control of endoplasmic reticulum (ER) stress].

\section{Discussion}

In the present study, we demonstrated that the expression of synoviolin and collagen secretion were enhanced by the overexpression of SP-C ${ }^{\text {exon4 } 4}$ in A549 cells and that collagen secretion was suppressed by the use of the synoviolin inhibitor, LS-102.
The pathogenic mechanisms of fibrosis have recently attracted the attention of researches, and studies have indicated that diverse factors, pathways and systems are involved in the progression of fibrosis. However, the detailed mechanisms involved are not yet fully understood. Wynn et al (8), Tanjore et al $(23,24)$ and Zhong et al $(25)$ reported that various pathways are involved in fibrosis, including the 
ER-stress pathway, and that ER stress leads to fibrosis. In the present study, we did not use any chemical or physical method to induce fibrosis, but instead we focused on a mutation in SP-C that was identified in mother-child cases of familial interstitial pneumonia, and which produced ER stress in A549 cells. Considering our results in the context of the dual role of synoviolin as an E3 ubiquitin ligase and collagen synthesis promoter, this study suggests the existence of a novel pathway: ER stress increases synoviolin synthesis, which promotes collagen synthesis, contributing to fibrosis. This result confirm the association between ER stress and fibrosis that has been previously reported $(23,25)$. Further studies are required to fully determine the association between synoviolin and fibrosis.

Excessive fibrosis affects various organs and tissues, including the endocardium, the heart, the liver, the kidneys, the skin, the bone marrow, the retroperitoneum and the lungs. Advanced fibrosis is a serious disorder that causes organ dysfunction and death; accordingly, antifibrotic drugs are in development. Various factors [TGF- $\beta, \mathrm{CTGF}$, tumor necrosis factor (TNF), endothelin, chemokines, chemokine receptors, and matrix metalloproteinases (MMPs)] and effector cells are involved in fibrosis $(19,20,26-28)$. As a result, there are several potential therapeutic vectors which may be used in the treatment of fibrosis, and thus a wide range of candidate therapeutic agents is available (8).

In the present study, we demonstrate that synoviolin expression and collagen secretion are enhanced by transfecting A549 cells with SP-C $\mathrm{C}^{\Delta \text { exon4 }}$ and that collagen secretion is suppressed by transfection with synoviolin siRNA or the synoviolin inhibitor, LS-102. Based on these findings and the recent studies indicating that synoviolin is involved in liver fibrosis (9) and renal fibrosis (10), our data suggest that the synoviolin inhibitor, LS-102 represents a candidate antifibrotic agent for the treatment of interstitial pneumonia; however, further studies, including in vivo studies, are required in order to confirm this therapeutic potential.

\section{Acknowledgements}

We thank the staff at the Future Medical Science at the Institute of Medical Science and the Integrated Gene Editing Section of Tokyo Medical University for their assistance. This study was funded in part by grants from the Naito Foundation, Natural Science Scholarship Daiichi-Sankyo Foundation of Life Science, Mitsubishi Tanabe Pharma Corporation, Bureau of Social Welfare and Public Health, The Ministry of Health Labour and Welfare, The Ministry of Education, Culture, Sports, Science and Technology, Japan Society for the Promotion of Science, Health Labour Sciences Research Grant, Takeda Science Foundation, AstraZeneca R\&D Grant 2013, Grant-in-Aid for Exploratory Research, Grant-in-Aid for Young Scientists (B).

\section{References}

1. Nogee LM, Dunbar AE III, Wert SE, Askin F, Hamvas A and Whitsett JA: A mutation in the surfactant protein $\mathrm{C}$ gene associated with familial interstitial lung disease. N Engl J Med 344: 573-579, 2001.

2. Beers MF and Mulugeta S: Surfactant protein $C$ biosynthesis and its emerging role in conformational lung disease. Annu Rev Physiol 67: 663-696, 2005.
3. Mulugeta S and Beers MF: Surfactant protein C: its unique properties and emerging immunomodulatory role in the lung. Microbes Infect 8: 2317-2323, 2006.

4. Nogee LM: Genetics of the hydrophobic surfactant proteins. Biochim Biophys Acta 1408: 323-333, 1998.

5. Mulugeta S, Nguyen V, Russo SJ, Muniswamy M and Beers MF: A surfactant protein C precursor protein BRICHOS domain mutation causes endoplasmic reticulum stress, proteasome dysfunction, and caspase 3 activation. Am J Respir Cell Mol Biol 32: 521-530, 2005.

6. Maguire JA, Mulugeta S and Beers MF: Endoplasmic reticulum stress induced by surfactant protein C BRICHOS mutants promotes proinflammatory signaling by epithelial cells. Am J Respir Cell Mol Biol 44: 404-414, 2011.

7. Wynn TA: Cellular and molecular mechanisms of fibrosis. $\mathbf{J}$ Pathol 214: 199-210, 2008.

8. Wynn TA and Ramalingam TR: Mechanisms of fibrosis: therapeutic translation for fibrotic disease. Nat Med 18: 1028-1040, 2012.

9. Hasegawa D, Fujii R, Yagishita N, et al: E3 ubiquitin ligase synoviolin is involved in liver fibrogenesis. PLoS One 5: e13590, 2010.

10. Li LY Shen, Ding Y, Liu Y, Su D and Liang X: Hrd1 participates in the regulation of collagen I synthesis in renal fibrosis. Mol Cell Biochem 386: 35-44, 2014.

11. Amano T, Yamasaki S, Yagishita N, et al: Synoviolin/Hrd1, an E3 ubiquitin ligase, as a novel pathogenic factor for arthropathy. Genes Dev 17: 2436-2449, 2003.

12. Kaneko M, Ishiguro M, Niinuma $Y$, Uesugi $M$ and Nomura $Y$ : Human HRD1 protects against ER stress-induced apoptosis through ER-associated degradation. FEBS Lett 532: 147-152, 2002.

13. Kaneko $M$ and Nomura Y: ER signaling in unfolded protein response. Life Sci 74: 199-205, 2003.

14. Wang WJ, Mulugeta S, Russo SJ, et al: Deletion of exon 4 from human surfactant protein $\mathrm{C}$ results in aggresome formation and generation of a dominant negative. J Cell Sci 116: 683-692, 2003.

15. Yamasaki S, Yagishita N, Sasaki T, et al: Cytoplasmic destruction of p53 by the endoplasmic reticulum-resident ubiquitin ligase 'Synoviolin'. EMBO J 26: 113-122, 2007.

16. Tsuchimochi K, Yagishita N, Yamasaki S, et al: Identification of a crucial site for synoviolin expression. Mol Cell Biol 25: 7344-7356, 2005.

17. Nogee LM, Dunbar AE III, Wert S, Askin F, Hamvas A and Whitsett JA: Mutations in the surfactant protein $\mathrm{C}$ gene associated with interstitial lung disease. Chest 121: S20S-S21, 2002.

18. Thomas AQ, Lane K, Phillips J III, et al: Heterozygosity for a surfactant protein $\mathrm{C}$ gene mutation associated with usual interstitial pneumonitis and cellular nonspecific interstitial pneumonitis in one kindred. Am J Respir Crit Care Med 165: 1322-138, 2002.

19. Border WA and Noble NA: Transforming growth factor beta in tissue fibrosis. N Engl J Med 331: 1286-1292, 1994.

20. Bonniaud P, Margetts PJ, Kolb M, Haberberger T, Kelly M, Robertson J and Gauldie J: Adenoviral gene transfer of connective tissue growth factor in the lung induces transient fibrosis. Am J Respir Crit Care Med 168: 770-778, 2003.

21. Myllyharju J: Prolyl 4-hydroxylases, the key enzymes of collagen biosynthesis. Matrix Biol 22: 15-24, 2003.

22. Yagishita N, Aratani S, Leach C, et al: RING-finger type E3 ubiquitin ligase inhibitors as novel candidates for the treatment of rheumatoid arthritis. Int J Mol Med 30: 1281-1286, 2012.

23. Tanjore H, Cheng DS, Degryse AL, Zoz DF, Abdolrasulnia R, Lawson WE and Blackwell TS: Alveolar epithelial cells undergo epithelial-to-mesenchymal transition in response to endoplasmic reticulum stress. J Biol Chem 286: 30972-30980, 2011.

24. Tanjore H, Lawson WE and Blackwell TS: Endoplasmic reticulum stress as a pro-fibrotic stimulus. Biochim Biophys Acta 1832: 940-947, 2013

25. Zhong Q, Zhou B, Ann DK, et al: Role of endoplasmic reticulum stress in epithelial-mesenchymal transition of alveolar epithelial cells: effects of misfolded surfactant protein. Am J Respir Cell Mol Biol 45: 498-509, 2011.

26. Hogaboam CM, Steinhauser ML, Chensue SW and Kunkel SL: Novel roles for chemokines and fibroblasts in interstitial fibrosis. Kidney Int 54: 2152-2159, 1998.

27. Arai M, Ikawa Y, Chujo S, et al: Chemokine receptors CCR2 and CX3CR1 regulate skin fibrosis in the mouse model of cytokineinduced systemic sclerosis. J Dermatol Sci 69: 250-258, 2013.

28. Fernandez IE and Eickelberg O: New cellular and molecular mechanisms of lung injury and fibrosis in idiopathic pulmonary fibrosis. Lancet 380: 680-688, 2012. 